\title{
Calculations of spin response functions in rings with Siberian Snakes and spin rotators
}

\author{
Yu. M. Shatunov \\ Budker Institute of Nuclear Physics, Novosibirsk, 630090, Russia \\ S. R. Mane* \\ Convergent Computing, Inc., P.O. Box 561, Shoreham, New York 11786, USA
}

(Received 10 November 2008; published 19 February 2009)

\begin{abstract}
The so-called spin response formalism, which is linear response theory applied to spin dynamics in storage rings, can calculate the resonance strengths for spin flippers in storage rings of arbitrary structure, including rings with Siberian Snakes and spin rotators. We calculate so-called spin response functions for a model of the RHIC lattice, for various scenarios of spin rotator settings.

DOI: 10.1103/PhysRevSTAB.12.024001

PACS numbers: 29.27.Hj, 29.20.D-, 02.60.Lj
\end{abstract}

\section{INTRODUCTION}

The program ASPIRRIN $[1,2]$ is an ideal tool to calculate spin flip resonance strengths in storage rings. ASPIRRIN implements the so-called "spin response formalism," which is a linear response theory applied to spin dynamics in storage rings. For example, in a recent paper Shatunov and Mane [3] used ASPIRRIN to successfully calculate the spin flip resonance strengths of stored polarized proton [4] and deuteron [5] beams, where the data was taken using a radial field rf dipole spin flipper at the COSY storage ring. For rf dipole spin flippers, the spin response formalism encapsulates the contributions to the spin flip resonance strength from both the direct spin kick and also from the spin-orbit coupling due to the induced coherent betatron oscillations, in so-called "spin response functions." Much of the initial experimental and theoretical work on spin flippers was carried out at the Budker Institute of Nuclear Physics. For example, a spin flipper was used in an experiment to compare the ratio of the anomalous magnetic moments of electrons and positrons counterrotating in the same storage ring [6]. This experiment set a world record for $C P T$ invariance in a lepton system at the time it was published. An early example of a spin response function was derived by Kondratenko [7], and a fit to the COSY deuteron data only (using a spin response function), was published by Kondratenko, Kondratenko, and Filatov [8]. ASPIRRIN calculates the spin response functions for rings of arbitrary structure, including rings which contain spin rotators and Siberian Snakes, such as RHIC. A Siberian Snake is theoretically defined as any device which rotates the spin of a particle by $180^{\circ}$ around an axis in the horizontal plane, but leaves the orbital motion unchanged. A spin rotator is similarly defined as any device which rotates a spin from the vertical direction to the horizontal plane, or vice versa, leaving the orbital motion unchanged. For example, in a recent paper Mane [9] has derived

\footnotetext{
*srmane@optonline.net
}

analytical expressions for the spin response function $F_{3}$ (to be defined later in this paper), for a smooth focusing model with pointlike $\delta$-function Snakes, and demonstrated that numerical calculations using ASPIRRIN match the analytical solutions. In particular, it is proposed to operate a new spin flipper at RHIC [10], consisting of two radial field rf dipoles in series, with ancillary hardware, in the presence of full strength Siberian Snakes. It is therefore an obvious application of ASPIRRIN to calculate the spin response functions, for radial field rf dipoles, for a model of the RHIC lattice, and we shall do so in this paper. We analyze various scenarios, such as longitudinal polarization at both the STAR and PHENIX interaction points of RHIC, or radial polarization at one of the interaction points, etc., and demonstrate how the spin response functions vary with the configuration of the spin rotators. Our calculations are performed only for the RHIC Blue ring; the results for the Yellow ring will be qualitatively similar but quantitatively different. Also, we treat only one optics setting, with fractional betatron tunes of $(0.7059,0.7148)$ and beta functions of approximately $40 \mathrm{~cm}$ (in both planes) at the STAR and PHENIX interaction points. Our calculations are intended to demonstrate the capabilities of ASPIRRIN and the spin response theory for rings with Siberian Snakes and spin rotators. However, RHIC has been operated with various optics settings, to optimize the luminosity and beam lifetime. Hence, for precise comparisons with measurements, the calculations must be performed with the precise machine optics in use at the time. It is beyond the scope of this paper to address such issues.

The structure of this paper is as follows. We present the basic theoretical formalism in Sec. II. We also briefly explain the peculiar features of spin flipping in rings with Siberian Snakes. The calculations for RHIC are presented in Sec. III, and Sec. IV concludes.

\section{BASIC THEORY}

All the symbols below have their usual meanings. The formalism below mainly follows [1]. We treat a particle of 
charge $e$, mass $m$, velocity $\boldsymbol{v}$, spin $\boldsymbol{s}$, and magnetic moment anomaly $G=\frac{1}{2}(g-2)$. The Lorentz factor is $\gamma=$ $1 / \sqrt{1-v^{2} / c^{2}}$. The reference momentum is $p_{0}=$ $m v_{0} \gamma_{0}$. We employ cgs units, so the Lorentz force is $e[\boldsymbol{E}+(\boldsymbol{v} / c) \times \boldsymbol{B}]$. We denote the magnetic rigidity by $B \rho=p_{0} c / e$. The arclength along the reference orbit is denoted by $s$. The independent variable in the equations of motion in the generalized azimuth is $\theta=s / R$, where the ring circumference is $2 \pi R$. The coordinate system is $(\hat{\boldsymbol{x}}, \hat{\boldsymbol{y}}, \hat{z})$, a right-handed orthogonal system where $\hat{\boldsymbol{x}}$ is radially outward, $\hat{\boldsymbol{y}}$ is along the reference orbit, and $\hat{z}=$ $\hat{\boldsymbol{x}} \times \hat{\boldsymbol{y}}$ (this is vertical in a planar ring). The positive sense of circulation is counterclockwise around the ring. Let $x$ and $z$ be the horizontal and vertical coordinates of a particle, and $p_{x}$ and $p_{z}$ are their conjugate momenta, respectively. The horizontal and vertical betatron tunes will be denoted by $\nu_{x}$ and $\nu_{z}$. The spin tune (the spin precession frequency in units of the beam circulation frequency) will be denoted by $\nu$. For future reference, we introduce here the periodic $\delta$ function:

$$
\delta_{p}\left(\theta-\theta_{0}\right)=\sum_{j=-\infty}^{\infty} \delta\left(\theta-\theta_{0}-2 j \pi\right) .
$$

We also define the dimensionless scaled magnetic fields on the reference orbit $K_{x, y, z}=B_{x, y, z} / B_{0}$, where $B_{0}$ is a reference value given by the arc dipoles, i.e., excluding Siberian Snakes and local bending magnets in straight section near an interaction point,

$$
B_{0}=\frac{1}{2 \pi} \int_{\operatorname{arcs}} B_{z} d \theta .
$$

For nonplanar rings the reference orbit curvatures are $K_{z}=$ $R / \rho_{x}$ and $K_{x}=-R / \rho_{z}$, where $\rho_{x}$ and $\rho_{z}$ are the bend radii in the horizontal and vertical planes, respectively.

In this paper, the unperturbed motion is based on the ideal reference orbit. The orbital variables $x, z, p_{x}$, etc., include all terms, i.e., closed orbit imperfections, and free and forced betatron oscillations around the reference orbit. We neglect electric fields, in particular, rf cavities and synchrotron oscillations. This is an acceptable approximation for RHIC.

We discuss briefly the neglect of electric fields. In the absence of electric fields, the effects of the magnetic fields in the spin precession equation of motion (the Thomas-Bargmann-Michel-Telegdi (BMT) equation $[11,12])$ can be completely expressed using $\left(x, x^{\prime}, z, z^{\prime}\right)$ and solenoidal fields. This was done, for example, by Courant and Ruth [13], leading to a compact formula for the strengths of imperfection and intrinsic depolarizing spin resonances. (Imperfection resonances are driven by closed orbit imperfections and intrinsic resonances are driven by the free vertical betatron oscillations.) The Courant-Ruth formula treats only planar uncoupled rings, i.e., without Siberian Snakes and spin rotators, and it also does not treat spin flippers. The spin response formalism treats transverse coupling, and full or partial Siberian Snakes and spin rotators, but again the orbital motion is purely in magnetic fields. However, to treat bunched beams, we require rf cavities and synchrotron oscillations. In that case, the spin precession vector in the Thomas-BMT equation cannot be expressed purely using $\left(x, x^{\prime}, z, z^{\prime}\right)$ and solenoidal fields. However, the SLIM formalism, developed by Chao [14], treats the spin-orbit coupling (to first order) including rf cavities and fully coupled six-dimensional orbital motion. Chao's original treatment did not include coherent orbital oscillations and spin flippers, but his formalism has recently been extended to do so by Barber [15]. Barber [16] has used his extended formalism to also calculate the spin flip resonance strengths for protons and deuterons at COSY. One of us (Mane) has checked with Barber to confirm that the ASPIRRIN calculations and Barber's results agree when both programs are applied to the same COSY optics file. Alternatively, the ASPIRRIN formalism could be modified to include rf cavities and synchrotron oscillations, which would lead to a basically equivalent formalism, although one would no longer be able to write such a succinct integral as Eq. (6) below. Another feature of bunched beams is the existence of synchrotron sideband resonances. The consequences of sidebands are different for $e^{+} e^{-}$and hadron storage rings. In $e^{+} e^{-}$storage rings, the spacing between the sidebands is greater than the resonance strength $\nu_{s} \gg \varepsilon$, where $\varepsilon$ denotes the spin flip resonance width and $\nu_{s}$ is the synchrotron tune. (Recall that the integrated field of a spin flipper, i.e., the value of $\varepsilon$, is under user control, so we can always satisfy the above inequality.) The range of the flipper frequency sweep must be such as to cross only one resonance (the parent resonance, because the sidebands are usually much weaker). In hadron rings, the opposite condition is true, $\varepsilon \gg \nu_{s}$ and the synchrotron oscillations are adiabatic to the spin precession. The range of the flipper frequency sweep in this case must be large enough to cross the spin tune spread of the beam. The main source of the spin tune spread is the beam energy spread. The spin tune spread, due to the beam energy spread, is given by

$$
\Delta \nu=\gamma \frac{\partial \nu}{\partial \gamma} \frac{\Delta \gamma}{\gamma}
$$

For a planar uncoupled ring, it is well known that $\nu=G \gamma$ and so $\gamma(\partial \nu / \partial \gamma)=G \gamma$, but a more detailed calculation is required for a ring with Siberian Snakes and spin rotators. It is beyond the scope of our paper to discuss such details. If the value of the synchrotron tune is small (as at RHIC), the ASPIRRIN formalism will still give a good approximation for the spin response functions.

The spin precession equation of motion in the externally prescribed electric and magnetic fields of the accelerator is known as the Thomas-BMT equation $[11,12]$. Using $\theta$ as 
the independent variable, the equation takes the form $d \boldsymbol{s} / d \theta=\boldsymbol{W} \times \boldsymbol{s}$. We subdivide the spin precession vector into two parts $\boldsymbol{W}=\boldsymbol{W}_{0}+\boldsymbol{w}$, where $\boldsymbol{W}_{0}$ is the spin precession vector on the ideal (reference) orbit, and $\boldsymbol{w}$ describes the off-axis motion. The spin motion due to $w$ is treated as a perturbation (this includes spin flippers). The unperturbed spin precession equation is $d \boldsymbol{s} / d \theta=\boldsymbol{W}_{0} \times \boldsymbol{s}$. It has three linearly independent solutions. One of these solutions, denoted by the unit vector $\boldsymbol{n}_{0}$, is periodic after one turn round the ring: $\boldsymbol{n}_{0}(\theta+2 \pi)=\boldsymbol{n}_{0}(\theta)$. Denote the two other linearly independent solutions on the reference orbit by the unit vectors $\boldsymbol{\eta}_{1}$ and $\boldsymbol{\eta}_{2}$, respectively, and let $\left\{\boldsymbol{n}_{0}, \boldsymbol{\eta}_{1}, \boldsymbol{\eta}_{2}\right\}$ be a right-handed orthonormal triad. The vectors $\boldsymbol{\eta}_{1}$ and $\boldsymbol{\eta}_{2}$ are not periodic after one turn. Define $\boldsymbol{\eta} \equiv \boldsymbol{\eta}_{1}-i \boldsymbol{\eta}_{2}$, then $\boldsymbol{\eta}(\theta+2 \pi)=e^{i 2 \pi \nu} \boldsymbol{\eta}(\theta)$. The normalization is $\left|\boldsymbol{n}_{0}\right|^{2}=1$ and $|\boldsymbol{\eta}|^{2}=2$.

The Froissart-Stora formula [17] for crossing an isolated spin resonance in a planar uncoupled ring is

$$
\frac{P_{f}}{P_{i}}=2 e^{-\pi|\varepsilon|^{2} /(2|\alpha|)}-1
$$

Here $P_{i}$ and $P_{f}$ are the initial and final asymptotic vertical polarizations, respectively. The value of $|\varepsilon|$ is called the resonance strength, and $\alpha=d \nu / d \theta$ is the rate of change of the spin tune, assumed constant. Equation (4) is derived assuming the resonance is crossed at a uniform rate, from far below to far above the resonant tune. Equation (4) has the property that if $|\varepsilon|^{2} /|\alpha| \gg 1$, then $P_{f} \simeq-P_{i}$, i.e., the (vertical) polarization is reversed with negligible loss of magnitude. This is called "adiabatic spin flip." The Froissart-Stora formula is also applicable to spin flippers. The spin tune is held fixed, and the (fractional part of the) flipper tune (or its mirror) is swept across the fractional part of the spin tune. Now $\alpha$ in Eq. (4) is $d \nu_{\text {rf }} / d \theta$, the rate of change of the spin flipper tune. For a successful spin flipper operation, the parameter values must be chosen to achieve adiabatic spin flip, to reverse the polarization with minimal loss of magnitude.

Many of the basic principles of spin flipping in rings with Siberian Snakes were worked out by Koop and Shatunov [18]. Here, we discuss some of the caveats for spin flipping in a ring with Siberian Snakes. In a ring with two diametrically opposed orthogonal Snakes, as at RHIC, the spin tune is $\nu=\frac{1}{2}$. Hence, the location of the spin flip resonance $\left[\nu_{\mathrm{rf}}\right]=\nu$ and the mirror resonance $1-\left[\nu_{\mathrm{rf}}\right]=$ $\nu$ coincide, where $\left[\nu_{\mathrm{rf}}\right]$ is the fractional flipper tune, so the condition of an isolated spin resonance is not satisfied. Hence, the Snakes should be detuned, to shift the spin tune slightly away from $\frac{1}{2}$, so that the spin flip resonance and its mirror are separated. The spin flipper tune is then swept across only one of the resonances. However, it is pointed out in [10] that for optimum luminosity and beam lifetime, RHIC has a limited ability to change the operating point in this way. Instead, the new RHIC spin flipper is designed so that the driving term of the mirror resonance is zero, so that the spin flipper can be operated with full strength Snakes and a spin tune of $\frac{1}{2}$.

Because the ratio of the peak integrated field of a spin flipper divided by $B \rho$ is small, it is adequate to solve the spin motion only to the first order in the orbital motion, to calculate spin flip resonance strengths. The details of the solution are given in [1]. The resonance strength, for a resonant spin tune $\nu_{\text {res }}$, is

$$
\begin{aligned}
\varepsilon= & \frac{1}{2 \pi} \int_{0}^{2 \pi}\left[\Delta K_{z} F_{1}-\Delta K_{x} F_{3}+(1\right. \\
& \left.+G) \Delta K_{y} \eta_{y}\right]_{\nu=\nu_{\mathrm{res}}} d \theta
\end{aligned}
$$

Here $\Delta K_{x, y, z}$ are (the resonant terms of) the perturbing fields along $x, y$, and $z$, respectively, e.g., due to closed orbit imperfections, or (in our case) spin flippers. The full set of "spin response functions" $F_{1}-F_{5}$ are defined in $[1,2]$. Equation (5) has an obvious interpretation that $F_{3}$ gives the "response" to a unit vertical momentum kick, with $\Delta K_{x}$ setting the actual scale of the overall perturbation. Only the function $F_{3}$ in Eq. (5) is relevant in this paper. For uncoupled orbital motion, the expression for $F_{3}$ is given by [1]

$$
\begin{aligned}
F_{3}\left(\theta_{0}\right)= & \frac{i}{2}\left\{\frac { f _ { z } ( \theta _ { 0 } ) } { e ^ { i 2 \pi ( \nu - \nu _ { z } ) } - 1 } \int _ { \theta _ { 0 } } ^ { \theta _ { 0 } + 2 \pi } \left[f_{z}^{* \prime}\left(\nu_{0}^{2}+G\right) K_{z} \eta_{y}\right.\right. \\
& \left.+f_{z}^{*}(1+G) K_{z}^{\prime} \eta_{y}\right] d \theta \\
& -\frac{f_{z}^{*}\left(\theta_{0}\right)}{e^{i 2 \pi\left(\nu+\nu_{z}\right)}-1} \int_{\theta_{0}}^{\theta_{0}+2 \pi}\left[f_{z}^{\prime}\left(\nu_{0}^{2}+G\right) K_{z} \eta_{y}\right. \\
& \left.\left.+f_{z}(1+G) K_{z}^{\prime} \eta_{y}\right] d \theta\right\} .
\end{aligned}
$$

Here $\nu_{0}=G \gamma$ and $f_{z}=\sqrt{\beta_{z} / R} e^{i \phi_{z}}$, where $d \phi_{z} / d \theta=$ $R / \beta_{z}$ and $\beta_{z}$ is the vertical beta function. Also $f_{z}^{\prime}=$ $d f / d \theta$ and $\nu=\frac{1}{2}$ in the resonance denominators. For the orbital motion, to leading order the Snakes and spin rotators at RHIC are "optically transparent" and are equivalent to drift spaces of the same length as the space they occupy. Hence, for the orbital motion, we model the Snakes and spin rotators as drift spaces, and for the spin motion we treat them as pointlike $\delta$-function rotations, with no effect on the off-axis spin motion. This is satisfactory if the orbit excursions in the Snakes and spin rotators are small. If the spin flipper is operated at low energies, where the orbit excursions are large, a more detailed analysis should be performed. The Snakes and spin rotators affect the value of $\eta_{y}$. Hence, the value of $F_{3}$ will depend on the configurations of the spin rotators at the interaction points.

\section{CALCULATIONS FOR RHIC}

In this section, we shall calculate the spin response function $F_{3}$ for RHIC, using ASPIRRIN, for various scenarios of the spin rotator settings. We shall perform our calculations at beam energies of 100, 205, and $250 \mathrm{GeV}$. 
From Eq. (6), $F_{3}=F_{3}(\theta, \nu)$ depends on the azimuth and on the spin tune, and $\nu=\frac{1}{2}$ at RHIC. The value of $F_{3}$ also depends on the ring optics and, in particular, on the vertical betatron tune, but we display only the parameters relevant for the analysis below. Let $j=1,2$ and let the kick angles for the orbital motion be $\varphi_{j}$ (here $\chi_{j}$ are initial phases):

$$
\varphi_{j}=-\frac{(B L)_{j}}{B \rho} \cos \left(\nu_{\mathrm{rf}} \theta+\chi_{j}\right) \delta_{p}\left(\theta-\theta_{j}\right)
$$

The minus sign is because a positive radial magnetic field kicks a positively charged particle downwards. Set $\nu_{\mathrm{rf}}=\frac{1}{2}$ and write $\varphi_{j}=\frac{1}{2}\left(\Delta K_{x j}^{+}+\Delta K_{x j}^{-}\right)$, where

$$
\Delta K_{x j}^{ \pm}=-\frac{1}{2} \frac{(B L)_{j}}{B \rho} e^{\mp i\left[(1 / 2) \theta_{j}+\chi_{j}\right]} \delta_{p}\left(\theta-\theta_{j}\right) .
$$

Substituting in Eq. (5), we get the resonance strength and mirror

$$
\begin{aligned}
\varepsilon_{ \pm}= & -\frac{1}{2 \pi} \sum_{j=1,2}\left[\Delta K_{x j}^{ \pm} F_{3}\left(\theta_{j}, \nu\right)\right]_{\nu=\nu_{\mathrm{rf}}=1 / 2} \\
= & F_{3}\left(\theta_{1}, \frac{1}{2}\right) \frac{(B L)_{1}}{4 \pi B \rho} e^{\mp i\left[(1 / 2) \theta_{1}+\chi_{1}\right]} \\
& +F_{3}\left(\theta_{2}, \frac{1}{2}\right) \frac{(B L)_{2}}{4 \pi B \rho} e^{\mp i\left[(1 / 2) \theta_{2}+\chi_{2}\right]}
\end{aligned}
$$

The key idea of the new spin flipper design for RHIC [10] is that the driving term of the mirror spin resonance is set to zero. This will allow spin flipping without detuning the Snakes. Hence, we demand $\varepsilon_{-}=0$. We solve for $(B L)_{2}$ in terms of $(B L)_{1}$. The answer is

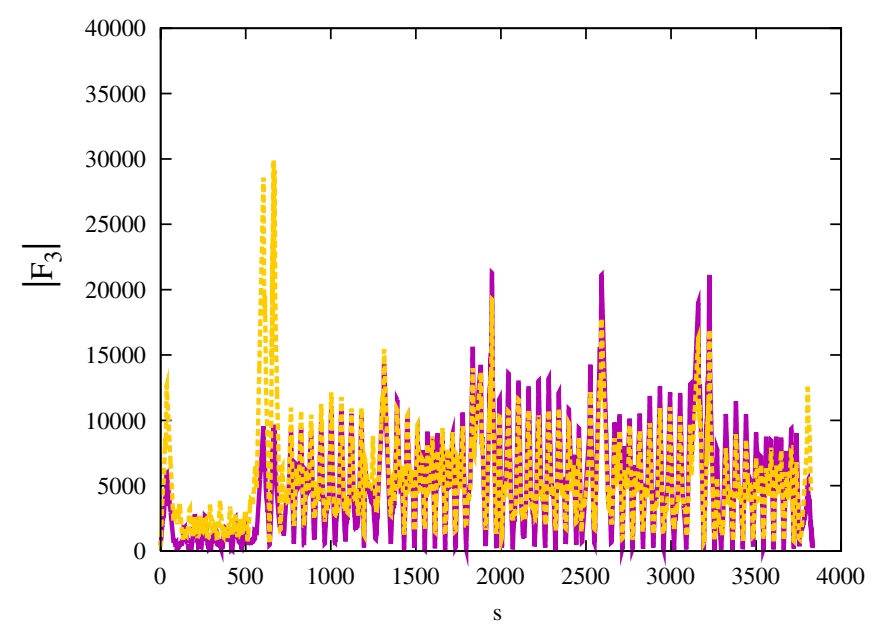

FIG. 1. (Color) Graph of $\left|F_{3}\right|$ around the circumference of RHIC. The origin is at 6 o'clock (STAR). The rotators are switched off, in this calculation. The solid curve is with Snakes on. The dashed curve is with Snakes off. The beam energy is slightly more than $100 \mathrm{GeV}$, so that the fractional spin tune is 0.5 in both curves.

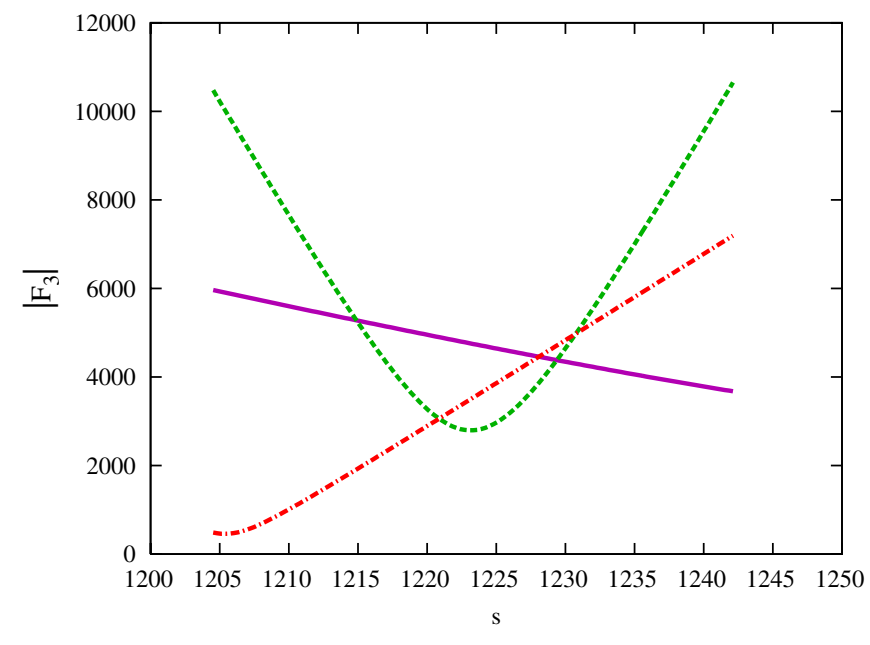

FIG. 2. (Color) Graph of $\left|F_{3}\right|$ at $100 \mathrm{GeV}$ in the drift space L232 of RHIC for three scenarios: (a) solid (purple) - no rotators; (b) dashed (green)-longitudinal polarization at both STAR and PHENIX; (c) dot-dashed (red)—no rotators at STAR, radial polarization at PHENIX. The horizontal axis shows the arclength in meters, measured from the 6 o'clock point.

$$
(B L)_{2}=-(B L)_{1} \frac{F_{3}\left(\theta_{1}, \frac{1}{2}\right)}{F_{3}\left(\theta_{2}, \frac{1}{2}\right)} e^{i\left(\chi_{1}-\chi_{2}\right)} e^{(i / 2)\left(\theta_{1}-\theta_{2}\right)} .
$$

There is a subtlety here: note that, by definition, $(B L)_{1}$ and $(B L)_{2}$ are real. However $F_{3}$ is in general complex. Since $\theta_{1}-\theta_{2}$ is fixed, this therefore imposes a constraint on $\chi_{1}-\chi_{2}$ that

$$
\Im\left\{\frac{F_{3}\left(\theta_{1}, \frac{1}{2}\right)}{F_{3}\left(\theta_{2}, \frac{1}{2}\right)} e^{i\left(\chi_{1}-\chi_{2}\right)} e^{(i / 2)\left(\theta_{1}-\theta_{2}\right)}\right\}=0 .
$$

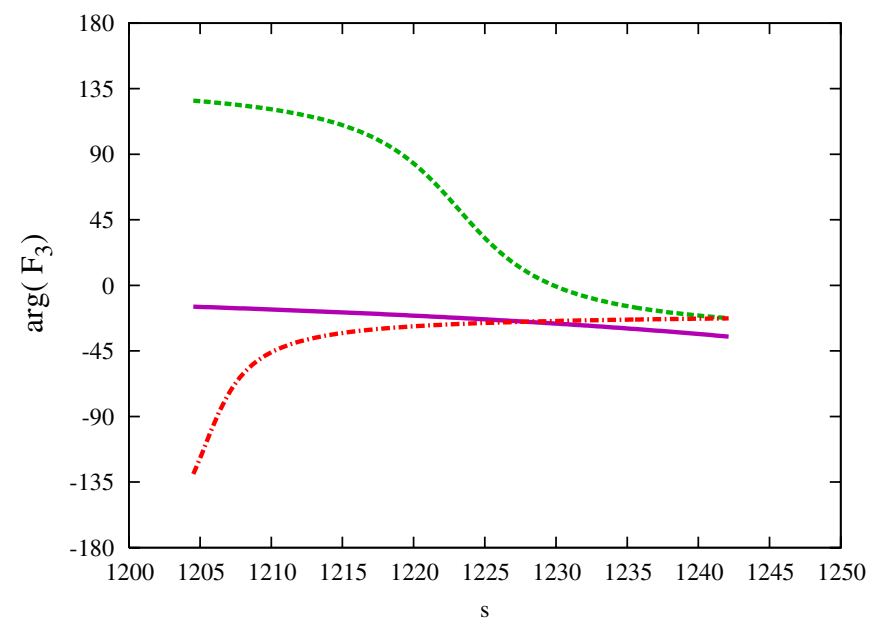

FIG. 3. (Color) Graph of $\arg \left(F_{3}\right)$ at $100 \mathrm{GeV}$ in the drift space L232 of RHIC for three scenarios: (a) solid (purple)—no rotators; (b) dashed (green)-longitudinal polarization at both STAR and PHENIX; (c) dot-dashed (red)—no rotators at STAR, radial polarization at PHENIX. The horizontal axis shows the arclength in meters, measured from the 6 o'clock point. 
Let us write $F_{3}\left(\theta_{j}\right)=\left|F_{3}\left(\theta_{j}\right)\right| e^{i \lambda_{j}}$. Then the constraint is

$$
\sin \left[\lambda_{1}-\lambda_{2}+\left(\chi_{1}-\chi_{2}\right)+\frac{1}{2}\left(\theta_{1}-\theta_{2}\right)\right]=0 .
$$

With this constraint, we have

$$
\begin{aligned}
\varepsilon_{+} & =\frac{(B L)_{1}}{4 \pi B \rho}\left[F_{3}\left(\theta_{1}, \frac{1}{2}\right) e^{-i\left[(1 / 2) \theta_{1}+\chi_{1}\right]}-\frac{F_{3}\left(\theta_{1}, \frac{1}{2}\right)}{F_{3}\left(\theta_{2}, \frac{1}{2}\right)} F_{3}\left(\theta_{2}, \frac{1}{2}\right) e^{i\left(\chi_{1}-\chi_{2}\right)} e^{(i / 2)\left(\theta_{1}-\theta_{2}\right)} e^{-i\left[(1 / 2) \theta_{2}+\chi_{2}\right]}\right] \\
& =\frac{(B L)_{1}}{4 \pi B \rho} F_{3}\left(\theta_{1}, \frac{1}{2}\right) e^{-i\left[(1 / 2) \theta_{1}+\chi_{1}\right]}\left\{1-e^{i\left[2\left(\chi_{1}-\chi_{2}\right)+\left(\theta_{1}-\theta_{2}\right)\right]}\right\}=\frac{(B L) 1}{4 \pi B \rho} F_{3}\left(\theta_{1}, \frac{1}{2}\right) e^{-i\left[(1 / 2) \theta_{1}+\chi_{1}\right]}\left\{1-e^{-i 2\left(\lambda_{1}-\lambda_{2}\right)}\right\} \\
& =i \frac{(B L)_{1}}{2 \pi B \rho} F_{3}\left(\theta_{1}, \frac{1}{2}\right) e^{-i\left[(1 / 2) \theta_{1}+\chi_{1}\right]} e^{-i\left(\lambda_{1}-\lambda_{2}\right)} \sin \left(\lambda_{1}-\lambda_{2}\right) .
\end{aligned}
$$

The magnitude of the resonance strength is

$$
\left|\varepsilon_{+}\right|=\frac{1}{2 \pi} \frac{(B L)_{1}}{B \rho}\left|F_{3}\left(\theta_{1}, \frac{1}{2}\right) \sin \left(\lambda_{1}-\lambda_{2}\right)\right| .
$$

The sine term vanishes if $\lambda_{1}-\lambda_{2}=0$ or $\pi$, and reaches a maximum value (of 1 ) when $\lambda_{1}-\lambda_{2}= \pm \frac{1}{2} \pi$.

First we calculate the value of $\left|F_{3}\right|$ around the RHIC circumference, with the rotators switched off, in Fig. 1. The solid curve is with the Snakes on, and the dashed curve is with the Snakes off. Since $G \gamma \simeq 191.1$ at $100 \mathrm{GeV}$ (and $\nu=\frac{1}{2}$ with Snakes), we set the energy slightly above $100 \mathrm{GeV}$ so that $G \gamma=191.5$. Then the fractional spin tune was 0.5 for both curves. The value of $\left|F_{3}\right|$ in Fig. 1 shows distinct peaks at the low-beta quadrupoles near the STAR and PHENIX interaction points (especially with Snakes off), but overall the two curves are of comparable magnitude. The use of Snakes does not reduce the value of $\left|F_{3}\right|$, in general. The analytical calculations in [9] show

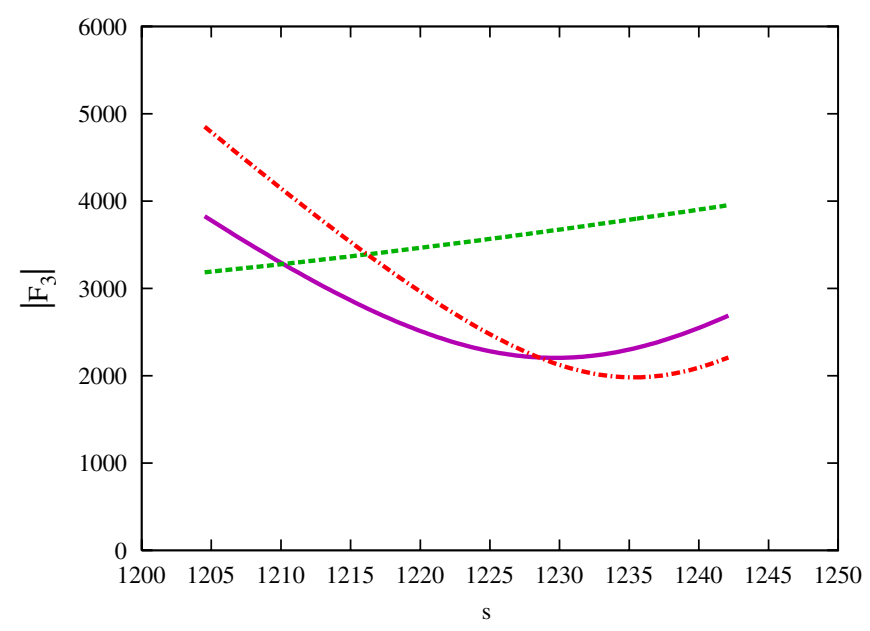

FIG. 4. (Color) Graph of $\left|F_{3}\right|$ at $205 \mathrm{GeV}$ in the drift space L232 of RHIC for three scenarios: (a) solid (purple)—no rotators; (b) dashed (green) - longitudinal polarization at both STAR and PHENIX; (c) dot-dashed (red)—no rotators at STAR, radial polarization at PHENIX. The horizontal axis shows the arclength in meters, measured from the 6 o'clock point. that it is possible for the value of $\left|F_{3}\right|$ to be larger in a ring with Snakes, than in the same model without Snakes.

It is proposed to locate the spin flipper in the L232 drift space of RHIC, so we calculate $F_{3}$ in that drift space. Obviously, the Snakes are switched on in the calculations below. We perform calculations at 100, 205, and $250 \mathrm{GeV}$, and at each energy we analyze three scenarios: (i) no rotators; (ii) longitudinal polarization at both STAR and PHENIX; (iii) vertical polarization (no rotators) at STAR, radial polarization at PHENIX. The value of $\left|F_{3}\right|$ at $100 \mathrm{GeV}$ for the above three scenarios is shown in Fig. 2. Next, we plot the value of $\arg \left(F_{3}\right)$, in degrees, for the same three scenarios, in Fig. 3. It is immediately obvious that both the magnitude and phase of $F_{3}$ depend strongly on the spin rotator configuration at each IP. For the case without rotators, the phase of $F_{3}$ varies only slightly (from $-14^{\circ}$ to $-33^{\circ}$ ) across the drift space. In the other two scenarios, the phase of $F_{3}$ varies significantly across the drift space.

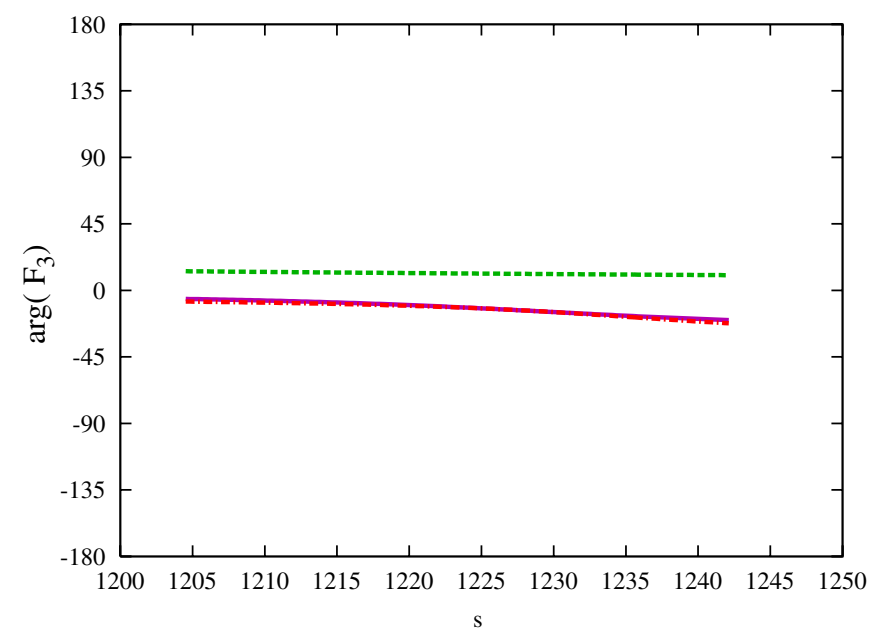

FIG. 5. (Color) Graph of $\arg \left(F_{3}\right)$ at $205 \mathrm{GeV}$ in the drift space L232 of RHIC for three scenarios: (a) solid (purple) - no rotators; (b) dashed (green)-longitudinal polarization at both STAR and PHENIX; (c) dot-dashed (red) — no rotators at STAR, radial polarization at PHENIX. The horizontal axis shows the arclength in meters, measured from the 6 o'clock point. 


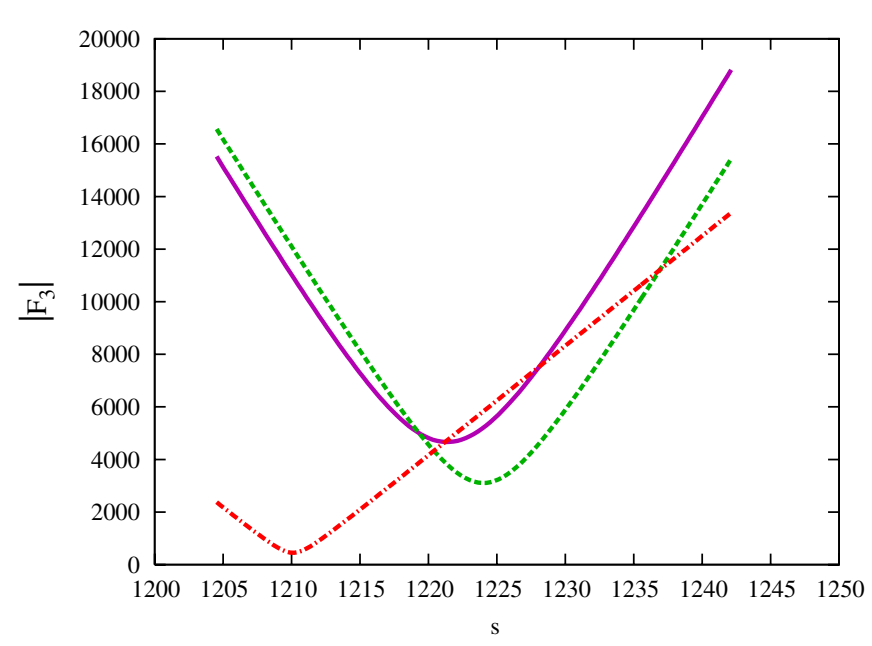

FIG. 6. (Color) Graph of $\left|F_{3}\right|$ at $250 \mathrm{GeV}$ in the drift space L232 of RHIC for three scenarios: (a) solid (purple)—no rotators; (b) dashed (green)-longitudinal polarization at both STAR and PHENIX; (c) dot-dashed (red)—no rotators at STAR, radial polarization at PHENIX. The horizontal axis shows the arclength in meters, measured from the 6 o'clock point.

Similarly, the value of $F_{3}$ at $205 \mathrm{GeV}$, for the same three scenarios, is shown in Figs. 4 and 5, for $\left|F_{3}\right|$ and $\arg \left(F_{3}\right)$, respectively. The corresponding graphs, for a beam energy of $250 \mathrm{GeV}$, are shown in Figs. 6 and 7. We see that the magnitude of $F_{3}$ does not increase appreciably from 100 to $205 \mathrm{GeV}$, and may even decrease, but $\left|F_{3}\right|$ is clearly larger at $250 \mathrm{GeV}$. We also see that, at both 205 and $250 \mathrm{GeV}$, the phase of $F_{3}$ changes relatively little across the drift space, for all three scenarios. This is significantly different from the behavior of the phase of $F_{3}$ at $100 \mathrm{GeV}$.

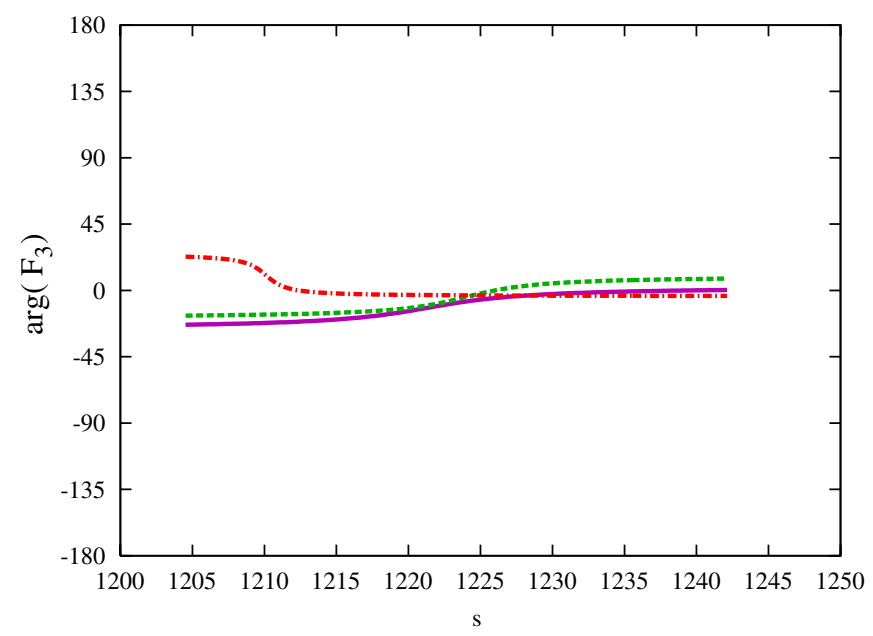

FIG. 7. (Color) Graph of $\arg \left(F_{3}\right)$ at $250 \mathrm{GeV}$ in the drift space L232 of RHIC for three scenarios: (a) solid (purple) - no rotators; (b) dashed (green) - longitudinal polarization at both STAR and PHENIX; (c) dot-dashed (red)—no rotators at STAR, radial polarization at PHENIX. The horizontal axis shows the arclength in meters, measured from the 6 o'clock point.

\section{CONCLUSION}

The program ASPIRRIN contains all of the required functionality to calculate spin flip resonance strengths in storage rings. The contributions of both the direct spin kick and of the induced coherent betatron oscillations (for $\mathrm{rf}$ dipoles) are encapsulated into so-called "spin response functions." ASPIRRIN calculates such functions for rings of arbitrary structure, including rings with spin rotators and Siberian Snakes, such as RHIC. The function $F_{3}$ describes the response to vertical momentum kicks, and is therefore relevant for radial field rf dipole spin flippers. We displayed calculations of $F_{3}$ for a model of the RHIC lattice, for various scenarios. We demonstrated, among other things, that the magnitude and phase of $F_{3}$ can depend significantly on the beam energy and the configuration of the spin rotators at the STAR and PHENIX interaction points of RHIC.

\section{ACKNOWLEDGMENTS}

We thank Dr. V. Ptitsyn for helpful information about ASPIRRIN.

[1] V. Ptitsyn, Ph.D. thesis, Budker Institute of Nuclear Physics, Novosibirsk, 1997 (in Russian).

[2] E. A. Perevedentsev, Yu. M. Shatunov, and V. Ptitsin, Proceedings of SPINO2, AIP Conf. Proc. No. 675 (American Institute of Physics, New York, 2003), p. 761.

[3] Yu. M. Shatunov and S. R. Mane, Phys. Rev. ST Accel. Beams 11, 094002 (2008).

[4] M. A. Leonova et al., Phys. Rev. ST Accel. Beams 9, 051001 (2006).

[5] A.D. Krisch et al., Phys. Rev. ST Accel. Beams 10, 071001 (2007).

[6] I. B. Vasserman et al., Phys. Lett. B 198, 302 (1987).

[7] A. M. Kondratenko, Novosibirsk Report No. 82-28, 1982.

[8] A. M. Kondratenko, M. A. Kondratenko, and Yu. N. Filatov, Pis'ma Fiz. Elem. Chastits At. Yadra 148, 902 (2008) [Phys. Part. Nucl. Lett. 5, 538 (2008)].

[9] S. R. Mane, Nucl. Instrum. Methods Phys. Res., Sect. A 600, 383 (2009).

[10] M. Bai and T. Roser, Phys. Rev. ST Accel. Beams 11, 091001 (2008).

[11] L. H. Thomas, Philos. Mag. 3, 1 (1927).

[12] V. Bargmann, L. Michel, and V.L. Telegdi, Phys. Rev. Lett. 2, 435 (1959).

[13] E.D. Courant and R.D. Ruth, Brookhaven National Laboratory Technical Report No. BNL 51270, 1980.

[14] A. W. Chao, Nucl. Instrum. Methods 180, 29 (1981).

[15] D. P. Barber, DESY Report No. 09-015, 2009.

[16] D. P. Barber (private communication).

[17] M. Froissart and R. Stora, Nucl. Instrum. Methods Phys. Res., Sect. A 7, 297 (1960) (in French).

[18] I. Koop and Yu. M. Shatunov, Proceedings of SPIN94, AIP Conf. Proc. No. 343 (American Institute of Physics, New York, 1995), pp. 317-320. 